\title{
MODELACIÓN DE LA CONTRIBUCIÓN ARBÓREA EN ANÁLISIS DE SUSCEPTIBILIDAD A DESLIZAMIENTOS SUPERFICIALES
}

\author{
ROBERTO J. MARÍN ${ }^{1}$ \\ JUAN PABLo OSORIO ${ }^{2}$
}

\section{RESUMEN}

En este artículo se realiza un análisis de susceptibilidad a ocurrencia de deslizamientos superficiales en un terreno montañoso tropical. Se emplea un método que permite modelar grandes áreas de terreno, cuantificando la contribución de los árboles a la estabilidad mediante tres parámetros principales: interceptación de lluvia, refuerzo aportado por las raíces y sobrecarga debida a su peso. Se utiliza un modelo de interceptación para determinar la lluvia disponible para infiltración y su distribución temporal durante la misma. Se describen los modelos hidrológicos de TRIGRS asociados a las condiciones iniciales de simulación, para estimar la variación de la presión de poros en profundidad. Esta variable se incluye en un modelo revisado de estabilidad de talud infinito, el cual considera los términos del refuerzo aportado por las raíces y la sobrecarga, evaluando la estabilidad en términos del factor de seguridad (FS) en toda una cuenca. Se simulan diferentes escenarios de densidad arbórea en una cuenca del Valle de Aburrá y se comparan los resultados con una modelación realizada sin considerar el efecto de los árboles en la estabilidad.

Los resultados obtenidos mostraron valores de FS < 1 (se predice falla con la ocurrencia del evento de lluvia simulado) en una zona delimitada por su nula presencia arbórea. El 26 de octubre de 2016, meses después de concluida la investigación y presentado el artículo a la Revista EIA, se desencadenó un deslizamiento superficial en la zona estudiada (municipio de Copacabana, deslizamiento en la Autopista Medellín-Bogotá K12+200, en la vereda El Cabuyal), lo cual indica que la metodología presentada puede ser una herramienta útil para la predicción de deslizamientos superficiales.

PALABRAS CLAVE: Estabilidad de taludes; Deslizamientos superficiales; TRIGRS; Interceptación de lluvia; Refuerzo de las raíces; Sobrecarga arbórea.

\section{MODELING TREE CONTRIBUTION IN SUSCEPTIBILITY ANALYSIS OF SHALLOW LANDSLIDES}

\section{ABSTRACT}

In this paper an assessment of shallow landslides susceptibility in a tropical and mountainous terrain is made. A method that allows to model slope stability over large areas is used. Tree contribution is quantified by means of three

1 Ingeniero Civil. GeoResearch International - GeoR, Escuela Ambiental, Facultad de Ingeniería, Universidad de Antioquia - UdeA.

2 Ingeniero Civil, PGDip, MBA, MEng, PhD, MIEI. GeoResearch International - GeoR, Escuela Ambiental, Facultad de Ingeniería, Universidad de Antioquia - UdeA.

Autor de correspondencia: Marín Sánchez R.J. (Roberto José): Universidad de Antioquia - UdeA; Calle 70 No. 52 21, Medellín, Colombia. Teléfonos: 4129419- 30152998 39 Correo electrónico: rjose.marin@udea.edu.co
Historia del artículo:

Artículo recibido: 8-VI-2016 / Aprobado: 30-X-2017

Disponible online: 3 de noviembre 2017

Discusión abierta hasta abril de 2019 
parameters: rainfall interception, root reinforcement and tree surcharge. A rainfall interception model is used to determine the rain available for infiltration and its temporal distribution during the simulation. The hydrological models included in TRIGRS are described, which allows to determine the pore water pressure depending on the initial conditions. This pore water pressure is used in the revised infinite slope stability model, along with root reinforcement and tree surcharge, in order to determine the slope stability in terms of a factor of safety (FS) for an entire basin. Different scenarios of tree density are modeled in a basin of the Valle de Aburra and the results are compared with a model performed without considering the effects of trees on the stability.

The results obtained showed values of FS $<1$ (predicting failure with the occurrence of the simulated rainfall event) in the delimited area due to the null presence of trees. On the 26th October 2016, months after the investigation was concluded and the paper submitted to Revista EIA, a shallow landslide occurred in the studied area (municipality of Copacabana, landslide on the Medellín-Bogotá Highway K12+200, in the El Cabuyal zone), indicating that the proposed methodology can be a useful tool for the prediction of shallow landslides.

KEYWORDS: Slope stability; Shallow landslides; TRIGRS; Rainfall interception; Root reinforcement; Tree surcharge.

\section{MODELAGEM DA CONTRIBUIÇÃO DE ÁRVORES NA ANÁLISE DA SUSCETIBILIDADE A DESLIZAMENTOS SUPERFICIAIS}

\section{RESUMO}

Utilizou-se um método para modelar a suscetibilidade à ocorrência de deslizamentos superficiais em grandes áreas de terra, quantificando a contribuição das árvores na estabilidade de taludes mediante três parâmetros principais: interceptação de chuva, o reforço fornecido pelas raízes e a sobrecarga devido ao seu peso. Foi usado um modelo de interceptação para determinar a infiltração da chuva disponível e sua distribuição temporal. Foram descritos os modelos hídricos com a utilização de TRIGRS, de acordo com as condições iniciais com o propósito de estimar a pressão dos poros. Esta variável foi incluída no modelo de estabilidade de taludes infinitos o qual considera os parâmetros reforço das raízes e sobrecarga, permitindo modelar a estabilidade em termos de segurança em toda uma bacia. Modelaram-se diferentes cenários de densidade de árvores em uma bacia do Valle de Aburrá e os resultados foram comparados com uma modelagem sem considerar o efeito das árvores na estabilidade.

Os resultados obtidos mostraram valores de FS <1 (prevê-se que falhar com a ocorrência de um evento de precipitação simulada) em uma área delimitada devido à presença nula de árvores. 026 de outubro de 2016 após conclusão da investigação e do documento enviado à Revista EIA, ocorre um deslizamento superficial de terra nessa área (município de Copacabana, na rodovia de Medellín-Bogotá K12 + 200, na zona El Cabuyal), podendo ser esta uma indicação de que a metodologia utilizada em esta investigação é uma ferramenta útil para a previsão de deslizamentos superficiais.

PALAVRAS-CHAVE: Estabilidade de taludes; Deslizamentos superficiais; TRIGRS; Interceptação de chuva; Reforço de raiz; Sobrecarga de árvore.

\section{INTRODUCCIÓN}

Los deslizamientos superficiales son un fenómeno de inestabilidad que afecta principalmente depósitos superficiales de espesor reducido, general- mente menores a $2 \mathrm{~m}$. Estos son desencadenados por eventos de lluvia de alta intensidad y/o de larga duración (Montrasio, Schilirò y Terrone, 2015). Si bien movilizan pequeños volúmenes de suelo, pueden distribuirse abundantemente a lo largo de grandes 
territorios, ocasionando muchas veces daños en cultivos, estructuras e incluso pérdidas de vidas humanas (Bordoni et al., 2015a; Bordoni et al., 2015b).

Existen diversos modelos físicos que pueden analizar la distribución espacial y temporal de deslizamientos superficiales a escala de cuenca, pero el éxito de estas modelaciones depende de una apropiada simulación de las características del terreno y de los mecanismos que desencadenan este fenómeno (Cascini, Cuomo y Della Sala, 2011). De esta manera, la predicción espacial y temporal de la ocurrencia de deslizamientos superficiales inducidos por lluvia es una difícil tarea; sin embargo, diferentes autores han adoptado diferentes métodos, entre los cuales se destacan (Raia et al., 2013; Salciarini et al., 2006):

1. Métodos empíricos (por ejemplo Godt, Baum y Chleborad, 2006; Guzzetti et al., 2008).

2. Métodos estadísticos (por ejemplo Coe et al., 2004; Crovelli, 2000; Guzzetti et al., 2006).

3. Métodos basados en los procesos físicos (por ejemplo Burton y Bathurst, 1998; Montgomery y Dietrich, 1994; Montrasio, Valentino y Losi, 2011; Pack, Tarboton y Goodwin, 1998; Wu y Sidle, 1995).

4. Combinación de los anteriores (por ejemplo Frattini, Crosta y Sosio, 2009; Raia et al., 2013).

Entre los factores que influencian los deslizamientos superficiales desencadenados por lluvias, las propiedades de la vegetación tienen una contribución importante. Los efectos de la vegetación en la estabilidad de laderas se pueden agrupar en dos tipos de mecanismos principales: hidrológicos y mecánicos. Hidrológicamente, la vegetación actúa reduciendo el agua disponible para infiltración y la humedad del suelo a través de la interceptación, evaporación y transpiración. Por otro lado, incrementa la capacidad de infiltración al aumentar la rugosidad del suelo y propiciar la generación de grietas de desecación. Mecánicamente, el anclaje de las raíces en estratos de suelo más estables puede beneficiar la estabilidad, así como el anclaje lateral en superficies susceptibles a fallar. De igual forma, las raíces pueden aumentar la resistencia cortante del terreno proporcionando una membrana de refuerzo a la capa del suelo. Por otra parte, los árboles constituyen una sobrecarga que incrementa los componentes de fuerza normales y paralelos a la ladera, lo que puede desfavorecer la estabilidad. Las fuerzas dinámicas transmitidas por el viento a través del tronco de los árboles también pueden representar un mecanismo adverso a la estabilidad (Gray y Sotir, 1996; Morgan y Rickson, 2003; Sidle y Ochiai, 2006; Stokes et al., 2008).

Si bien los efectos de la vegetación en la estabilidad de laderas han sido ampliamente estudiados alrededor del mundo, se han realizado pocas aproximaciones a nivel de cuenca o a escala regional que evalúen cuantitativamente la contribución de los árboles asociada a la susceptibilidad de ocurrencia de movimientos en masa (Kim et al., 2013). El presente artículo propone un método para evaluar la susceptibilidad a ocurrencia de deslizamientos superficiales inducidos por lluvia, en grandes áreas de terreno (a escala de cuenca hidrográfica), cuantificando el efecto de los árboles a partir de tres parámetros principales: interceptación de lluvia, refuerzo aportado por las raíces y sobrecarga debida al peso de los árboles.

\section{DESCRIPCIÓN GENERAL DEL MÉTODO}

El análisis de la estabilidad en la cuenca a evaluar se representa mediante el factor de seguridad (FS). Por medio de las herramientas disponibles con el uso de un sistema de información geográfica se elaboran, adecúan e introducen los datos de entrada, los cuales pueden ser variables con valores únicos en toda la cuenca o que se presentan en forma de mapas, representando su variabilidad espacial. Por medio de una configuración de celdas, los cálculos de presión de poros y del factor de seguridad se obtienen para cada una de ellas.

Para incorporar los efectos de los árboles en la estabilidad se implementa un método que acopla modelos existentes (modelos de interceptación, 
hidrológico y geotécnico). De esta manera, se utiliza el modelo de interceptación de lluvia de Liu (1997), el cual estima la lluvia disponible para infiltración, reduciendo la cantidad que es interceptada y evaporada desde el dosel arbóreo. Seguidamente, se emplea un modelo de infiltración transitoria para calcular la variación de la presión de poros durante el evento de lluvia simulado; en este caso se empleó el componente hidrológico de TRIGRS 2.0 (Baum, Savage y Godt, 2008). Finalmente, se utiliza el modelo revisado de estabilidad de taludes infinitos, propuesto por Kim et al. (2013), para determinar el factor de seguridad en cada celda, cuantificando la sobrecarga y el refuerzo aportado por las raíces de los árboles. A continuación se muestran con más detalle los modelos implementados.

\section{lluvia}

\subsection{Modelo de interceptación de}

El modelo de interceptación de Liu (1997) se basa en la utilización de parámetros físicos para estimar la interceptación de lluvia, de acuerdo a la siguiente ecuación:

$$
I=C_{m} *\left(D_{i}-D\right)\left[1-\left(1-b_{0}\right) * \frac{\bar{e}}{\bar{R}}\right]+\bar{e} * T,
$$

donde $C_{m}$ es la capacidad de almacenamiento del dosel, $D_{i}$ el índice de sequedad del dosel antes de la lluvia, $D$ el índice de sequedad del dosel, $b_{0}$ el coeficiente de escurrimiento libre, $\bar{e}$ la tasa de evaporación promedio del dosel húmedo, $\bar{R}$ la intensidad promedio y $T$ el periodo de tiempo.

En el presente estudio, la Ecuación (1) se aplica para cada intervalo de tiempo (al que se le asocia un valor de precipitación o intensidad promedio) que compone el evento de lluvia, obteniendo el índice de sequedad del dosel de cada periodo de acuerdo a la ecuación que se muestra a continuación, iniciando con un valor de $D_{i}=1$, lo que asume un dosel seco antes del evento de lluvia:

$$
D=D_{i} * \exp \left(-\frac{k P}{C_{m}}\right)
$$

donde $k=1-b_{0}$ es la cobertura del suelo y $P$ la precipitación promedio en el periodo de tiempo.

\subsection{Modelación de deslizamientos superficiales}

TRIGRS es un software diseñado para modelar la distribución espacial y temporal de deslizamientos superficiales inducidos por lluvia. El programa calcula los cambios de presión de poros y determina la variabilidad del factor de seguridad, debidos a la infiltración de la lluvia (Baum, Savage y Godt, 2008). Este programa acopla un modelo hidrológico que simula la infiltración de la lluvia con un modelo de estabilidad de taludes infinitos, el cual representa la estabilidad de celdas individuales por medio del factor de seguridad. El método de análisis de susceptibilidad a ocurrencia de deslizamientos de la presente investigación adopta únicamente el modelo hidrológico de TRIGRS 2.0, mientras que el componente geotécnico (es decir, una simulación aplicando todo el modelo de TRIGRS) se emplea para comparar los resultados obtenidos.

\subsection{Modelo hidrológico}

El modelo asume flujo en suelo homogéneo e isotrópico y se modela únicamente infiltración unidimensional vertical. TRIGRS 2.0 incluye modelos para condiciones iniciales saturadas y no saturadas. Para condiciones saturadas, los modelos de infiltración son basados en la solución lineal de la ecuación de Richards (1931), propuesta por Iverson (2000). Esta solución está compuesta por un componente estacionario y otro transitorio. Para el cálculo de presión de poros el programa permite una solución para el caso de frontera basal de profundidad infinita y para profundidad finita, las cuales representan diferentes condiciones bajo la superficie. La primera aplica donde las condiciones hidráulicas son uniformes en la profundidad, mientras que la segunda representa el caso en el cual se tiene una reducción considerable de la conductividad hidráulica en una profundidad finita. 
La solución generalizada para frontera basal de profundidad infinita está dada por la Ecuación (3):

$$
\begin{gathered}
\psi(Z, t)=(Z-d) \beta+2 \sum_{n=1}^{N} \frac{I_{n Z}}{K_{s}}=\left\{H ( t - t _ { n } ) \left[D_{1}\right.\right. \\
\left.\left.\left(t-t_{n}\right)\right]^{1 / 2} \operatorname{ierfc}\left[\frac{Z}{2\left[D_{1}\left(t-t_{n}\right)^{1 / 2}\right.}\right]\right\}-2 \sum_{n=1}^{N} \frac{I_{n Z}}{K_{s}}\{H \\
\left.\left(t-t_{n+1}\right)\left[D_{1}\left(t-t_{n+1}\right)\right]^{1 / 2} \operatorname{ierfc}\left[\frac{Z}{2\left[D_{1}\left(t-t_{n+1}\right)^{1 / 2}\right.}\right]\right\},
\end{gathered}
$$

donde $\psi$ es la presión de poros (en unidades de cabeza de presión); $t$ el tiempo; $\operatorname{con} Z=z / \cos \delta$, siendo $Z$ la coordenada en dirección vertical (positiva hacia abajo) y la profundidad bajo la superficie del suelo, $z$ la coordenada en dirección normal al talud (positiva hacia abajo), y $\delta$ es el ángulo del talud; $d$ es la profundidad en estado estacionario del nivel freático medido en dirección vertical.

Asimismo, $\beta=\cos ^{2} \delta-\left(I_{Z L T} / K_{s}\right)$, siendo $K_{s}$ la conductividad hidráulica saturada en la dirección $Z, I_{Z L T}$ la tasa de infiltración estacionaria (inicial) en la superficie, $I_{n Z}$ la tasa de infiltración a una intensidad dada para el n-ésimo intervalo de tiempo.

A su vez, $D_{1}=D_{0} / \cos ^{2} \delta$, siendo $D_{0}$ la difusividad hidráulica saturada $\left(D_{0}=K_{s} / S_{s^{\prime}}\right.$ donde $S_{s}$ es el almacenamiento especifico); $N$ el número total de intervalos. Los términos $H\left(t-t_{n}\right)$ representan la función de paso de Heaviside, donde $t_{n}$ es el tiempo en el n-ésimo intervalo en la secuencia de infiltración de lluvia.

La función ierfc tiene la forma

$$
\operatorname{iercf}(\eta)=\frac{1}{\sqrt{\pi}} \exp \left(-\eta^{2}\right)-\eta \operatorname{erfc}(\eta),
$$

donde $\operatorname{iercf}(\eta)$ es la función de error complementario.

Por su parte, la solución para presión de poros en el caso de frontera basal impermeable, para una profundidad finita $d_{L Z^{\prime}}$ se ilustra en la Ecuación (5), donde se adopta la misma notación de la Ecuación (3) y el término $d_{L Z}$ es la profundidad de la frontera basal impermeable, medida en la dirección $Z$.

$$
\begin{aligned}
& \psi(Z, t)=(Z-d) \beta \\
& +2 \sum_{n=1}^{N} \frac{I_{n Z}}{K_{s}} H\left(t-t_{n}\right)\left[D_{1}\left(t-t_{n}\right)\right]^{1 / 2} \sum_{m=1}^{N} \\
& \left\{\begin{array}{c}
\operatorname{ierfc}\left[\frac{(2 m-1) d_{L Z}-\left(d_{L Z}-Z\right)}{2\left[D_{1}\left(t-t_{n}\right)\right]^{1 / 2}}\right] \\
+\operatorname{ierfc}\left[\frac{(2 m-1) d_{L Z}-\left(d_{L Z}-Z\right)}{2\left[D_{1}\left(t-t_{n}\right)\right]^{1 / 2}}\right]
\end{array}\right\} \\
& -2 \sum_{n=1}^{N} \frac{I_{n Z}}{K_{s}} H\left(t-t_{n+1}\right)\left[D_{1}\left(t-t_{n+1}\right)\right]^{1 / 2} \sum_{m=1}^{N} \\
& \left\{\begin{array}{l}
\operatorname{ierfc}\left[\frac{(2 m-1) d_{L Z}-\left(d_{L Z}-Z\right)}{2\left[D_{1}\left(t-t_{n+1}\right)\right]^{1 / 2}}\right] \\
+\operatorname{ierfc}\left[\frac{(2 m-1) d_{L Z}-\left(d_{L Z}-Z\right)}{2\left[D_{1}\left(t-t_{n+1}\right)\right]^{1 / 2}}\right]
\end{array}\right\}
\end{aligned}
$$

Así mismo, el programa permite utilizar una solución analítica para flujo no saturado para la estimación de la infiltración. En esta condición se analiza el suelo como un sistema de dos capas, en donde una zona saturada con una franja capilar encima del nivel freático limita con una zona no saturada que se extiende hasta la superficie del suelo. Para esto, TRIGRS utiliza una transformación de coordenadas descrita por Iverson (2000) de la solución analítica unidimensional de la ecuación de Richards (1931), que describe la infiltración y el flujo vertical a través de la zona no saturada, como se muestra a continuación:

$$
\frac{\partial \theta}{\partial t}=\frac{\partial}{\partial Z}\left[K(\psi)\left(\frac{1}{\cos ^{2} \delta} \frac{\partial}{\partial Z}-1\right)\right],
$$

con $K(\psi)=K_{s} \exp \left(\alpha \psi^{*}\right)$, siendo $\psi$ la cabeza de presión, con $\psi^{*}=\psi-\psi_{0}$, siendo $\psi_{0}$ una constante $\left(\psi_{0}=0\right.$ o $\left.\psi_{0}=-1 / \alpha\right) ; K(\psi)$ una función de conductividad hidráulica, $K_{s}$ la conductividad hidráulica saturada; $\operatorname{con} \theta=\theta_{r}+\left(\theta_{s}-\theta_{r}\right) \exp \left(\alpha \psi^{*}\right)$, siendo $\theta$ el contenido volumétrico de agua, $\theta_{r}$ el contenido volumétrico de agua residual, $\theta_{s}$ el contenido volumétrico de agua en saturación y $\alpha$ un parámetro que se obtiene ajustando $K(\psi)$ a una curva característica del suelo. 


\subsubsection{Modelo geotécnico empleado en TRIGRS}

TRIGRS modela la estabilidad de las laderas mediante un análisis de estabilidad de talud infinito unidimensional, de acuerdo a Taylor (1948). En este análisis, la falla en un talud infinito se caracteriza por la relación entre las fuerzas resistentes de fricción con las fuerzas desestabilizadoras que son inducidas gravitacionalmente. Esta relación representa el factor de seguridad, el cual se calcula a una profundidad $Z$, como se muestra a continuación:

$$
F S(Z, t)=\frac{\tan \phi^{\prime}}{\tan \delta}+\frac{c^{\prime}-\psi(Z, t) Y_{w} \tan \phi^{\prime}}{\gamma_{s} Z \operatorname{sen} \delta \cos \delta},
$$

donde $c^{\prime}$ es la cohesión efectiva del suelo, $\phi^{\prime}$ el ángulo de fricción efectivo, $Y_{w}$ el peso específico del agua, $Y_{s}$ el peso específico del suelo, $\psi(Z, t)$ la presión de poros (en unidades de cabeza de presión). FS se calcula para presiones de poros a diferentes profundidades del suelo $Z$. Se predice falla cuando $F S<1$ y estabilidad cuando $F S \geq 1$, con equilibrio límite en $F S=1$. Ya que TRIGRS permite calcular FS a diferentes profundidades, la profundidad de iniciación del deslizamiento se predice en la mínima profundidad en la cual FS es menor que 1.

En Baum, Savage y Godt (2008), se tiene información adicional que describe de forma detallada tanto los modelos hidrológicos como el modelo geotécnico que se adoptan en el programa.

\subsection{Modelo revisado de estabilidad} de taludes infinitos de Kim et al. (2013)

Kim et al. (2013) integran un modelo hidrológico (Baum, Savage y Godt, 2002), un modelo de interceptación de lluvia (Rutter et al., 1971; Rutter, Morton y Robins, 1975) y una modificación a un modelo de estabilidad de taludes infinitos (Hammond et al., 1992), el cual cuantifica el aporte de los árboles a la estabilidad de taludes para analizar la susceptibilidad de grandes áreas de terreno a deslizamientos superficiales. La ecuación modificada de FS, la cual incluye los términos de refuerzo de las raíces y sobrecarga arbórea, permite integrar los cambios espaciales y temporales de la presión de poros modelados mediante TRIGRS, como se muestra en la siguiente expresión:

$F S(Z, t)=\frac{c_{r}+c^{\prime}+\left\{m_{t} \cos ^{2} \delta+\gamma_{s} Z \cos ^{2} \delta-\psi(Z, t) \gamma_{w}\right\} \tan \phi^{\prime}}{\left(m_{t}+\gamma_{s} Z\right) \operatorname{sen} \delta \cos \delta}$,

en donde $c_{r}$ es el refuerzo aportado por las raíces de los árboles, $c$ ' la cohesión efectiva, $m_{t}$ la sobrecarga arbórea, $\gamma_{s}$ el peso específico del suelo, $Z$ la profundidad del suelo, $\delta$ el ángulo del terreno, $\psi(Z, t)$ la presión de poros en función del tiempo y de la profundidad del suelo, $\gamma_{W}$ el peso específico del agua y $\phi^{\prime}$ el ángulo de fricción efectivo del suelo.

De esta manera, el método propuesto acopla el modelo de interceptación de lluvia de Liu (1997), empleado para determinar la lluvia disponible para infiltración que se utilizará en la simulación con uno de los modelos hidrológicos de TRIGRS 2.0, el cual se selecciona de acuerdo a las condiciones iniciales de frontera y saturación. De la modelación hidrológica se obtienen los cambios espaciales y temporales de la presión de poros y este término se incluye en la ecuación de FS del modelo revisado de estabilidad de taludes infinitos de Kim et al. (2013), la cual incluye los términos de refuerzo aportado por las raíces de los árboles y la sobrecarga. El método propuesto se prueba simulando diferentes escenarios de densidad arbórea sobre una cuenca y comparando los resultados con una modelación realizada en TRIGRS 2.0, sin incluir los efectos de los árboles en la estabilidad.

\section{CASO DE ESTUDIO}

\subsection{Sitio de estudio}

Se estudia una pequeña cuenca del Valle de Aburrá, subregión del departamento de Antioquia (Colombia). Está ubicada en la vereda El Cabuyal, en el municipio de Copacabana. Tiene un área de $2,94 \mathrm{~km}^{2}$ y un rango de elevaciones de $1674 \mathrm{~m}$ en la salida de la cuenca y $2554 \mathrm{~m}$ en la parte superior de la divisoria de aguas. La precipitación promedio es de 1875 mm/año, con pluviosidad relativamente 
constante. La temperatura media es de $22,5^{\circ} \mathrm{C}$. Los tipos de suelo de la vereda El Cabuyal se encuentran clasificados en superficies aluviales, montañas y suelos coluviales (ISEA Ltda, 2006).

\subsection{Condiciones geotécnicas e hidrológicas}

La modelación se realizó con una tormenta de diseño de 4 horas de duración. Este evento de lluvia se elaboró a partir de curvas IDF definidas para la zona de estudio (Smith y Vélez, 1997). El hietograma fue construido mediante el método de los bloques alternos (Chow, Maidment y Mays, 1988), eligiendo un periodo de retorno de 750 años. La lluvia obtenida tiene una precipitación acumulada de 95,7 mm distribuidos en 8 intervalos de 30 minutos, cada uno con determinada intensidad promedio (Figura 1).

Figura 1. Hietograma sintético del evento de lluvia a modelar. Se muestra la precipitación $(\mathrm{mm})$ en intervalos de 30 minutos y la precipitación acumulada $(\mathrm{mm})$.

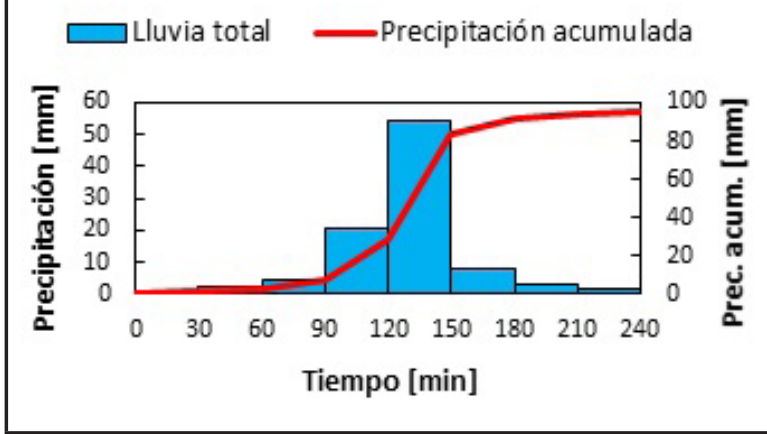

Para las simulaciones, se generaron mapas digitales del terreno con una resolución espacial de 10 m x 10 m. Utilizando ArcGIS, se elaboraron un modelo digital de elevación (Figura 2), un mapa de direcciones de flujo y un mapa de pendientes del terreno.

Marín y Castro (2015) analizaron la susceptibilidad a deslizamientos superficiales en esta misma cuenca, implementando el modelo de infiltración de TRIGRS 2.0, en condiciones saturadas, adoptando un valor constante de profundidad del suelo y definiendo la altura del nivel freático en esta misma profundidad. Así mismo, definieron las variables geotécnicas $\left(c^{\prime}, \phi^{\prime}, \gamma_{s}\right)$ e hidrológicas $\left(K_{s^{\prime}} D_{0}, K_{s^{\prime}}, \theta_{s^{\prime}}, \theta_{r^{\prime}}\right.$
$I_{Z L T}$ ) de acuerdo a valores encontrados en la literatura para el tipo de suelo encontrado en la zona.

Figura 2. Ubicación y modelo digital de elevación de la cuenca

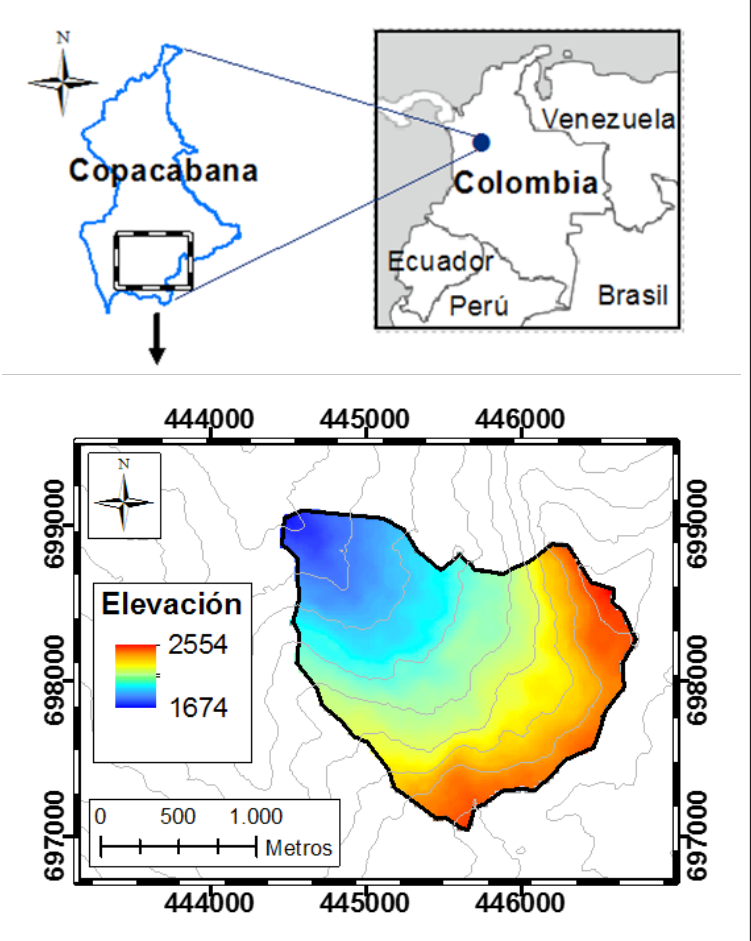

Debido a que en muchos casos existen limitaciones para obtener suficiente información de las condiciones del suelo, en otras modelaciones con TRIGRS se ha definido la profundidad de la superficie deslizante (asumida como la profundidad del suelo o frontera entre depósitos superficiales y roca madre) constante (Liao et al., 2011; Montrasio, Valentino y Losi, 2011; Park, Nikhil y Lee, 2013; Vieira y Fernandes, 2010), generalmente con valores entre $1 \mathrm{~m}$ y $2 \mathrm{~m}$, ya que la mayoría de los deslizamientos superficiales se producen entre estas profundidades (Meisina y Scarabelli, 2007). Por su parte, otros autores que han empleado TRIGRS han optado por utilizar funciones exponenciales que relacionan esta frontera con el ángulo del talud (Baum, Godt y Savage, 2010; Salciarini et al., 2006).

La presente modelación considera la profundidad del suelo con un límite inferior de $1,5 \mathrm{~m} \mathrm{y}$ uno superior de 1,8 m, implementando la función 
exponencial utilizada por Baum, Godt y Savage (2010), en donde la profundidad de la frontera basal impermeable $d_{L Z}$ está en función del ángulo del talud $(\delta), d_{L Z}=5,0 \exp (-0,04 \delta)$.

Por su parte, la profundidad del nivel freático previo a la tormenta la han simulado en el mismo nivel (Kim et al., 2013; Kim et al., 2010; Montrasio, Valentino y Losi, 2011; Park, Nikhil y Lee, 2013; Vieira y Fernandes, 2010) o como una fracción (Liao et al., 2011; Raia et al., 2013; Salciarini et al., 2006) de la profundidad del suelo. En el presente estudio, se simula la profundidad inicial del nivel freático $(d)$ como una fracción de esta frontera basal impermeable. Así, d = 0,75d $d_{L Z}$

Ya que a escala de cuenca no es fácil contar con información precisa de la variabilidad espacial de las propiedades del suelo, en este tipo de modelaciones se suele asignar valores promedio para muchos de los parámetros geotécnicos e hidrológicos. En este caso se definieron las variables requeridas a partir de información tomada de estudios geotécnicos realizados en esta cuenca, facilitados por otros investigadores, y de valores tomados de la literatura para el tipo de suelo encontrado en la zona. Tales estudios de campo constaron de 29 sondeos exploratorios en los cuales se determinaron la profundidad del nivel freático y, por medio de pruebas de laboratorio, se obtuvieron parámetros geotécnicos, de humedad y resistencia del suelo. Con base en esto, se asignó un valor de cohesión efectiva de $9 \mathrm{kN} /$ $\mathrm{m}^{2}$, un ángulo de fricción efectiva de $24^{\circ}$ y un peso específico del suelo de $15 \mathrm{kN} / \mathrm{m}^{3}$, constantes en toda la cuenca. Por su parte, la simulación de las variables hidrológicas se basó en la dependencia (correlación) existente entre algunas de estas variables (Raia et al., 2013), los estudios de campo y laboratorio mencionados, y una búsqueda en la literatura.

De esta manera, se determinó un valor de 5,0 $\mathrm{x} 10^{-5} \mathrm{~m} / \mathrm{s}$ para la conductividad hidráulica saturada $\left(K_{s}\right)$. Algunos autores han establecido el término de difusividad hidráulica $\left(D_{0}\right)$ como un múltiplo de la conductividad (Baum, Godt y Coe, 2011; Baum, Godt y Savage, 2010; Bordoni et al., 2015b). Park, Nikhil y
Lee (2013) y Liu y Wu (2008) afirman que $D_{0}$ suele estar entre 10 y 500 veces del valor de $K_{s}$; en ambas investigaciones adoptan un valor de $D_{0}=200 K_{s^{\prime}}$ En esta investigación, se toma esta relación, por lo que $D_{0}=1,0 \times 10^{-5} \mathrm{~m}^{2} / \mathrm{s}$. Asimismo, ambas investigaciones definieron la tasa de infiltración inicial como $I_{Z L T}=0,01 K_{s^{\prime}}$ Para esta investigación se adopta esta expresión, de tal manera que $I_{Z L T}=5,0 \times 10^{-7} \mathrm{~m} / \mathrm{s}$. Finalmente, se establecen el contenido de agua saturada $\left(\theta_{s}\right)$ y residual $\left(\theta_{r}\right)$ con valores de 0,45 y 0,1 , respectivamente, y al parámetro $\alpha$, el cual se aproxima a la inversa de la altura de la franja capilar sobre el nivel freático, se le asigna un valor de $1 \mathrm{~m}^{-1}$, por lo cual la simulación supone una franja capilar de gran espesor.

Los valores que se asignan a cada uno de los parámetros requeridos en la modelación de la presión de poros y del modelo geotécnico de TRIGRS 2.0 se resumen en la Tabla 1. Aunque algunas de las variables se modelan como valores constantes en toda la cuenca, cada una de ellas se puede incluir en la modelación como un parámetro variable espacialmente. Cabe destacar que esta modelación utiliza la solución de infiltración para flujo no saturado, de modo que la condición inicial simula un estrato de suelo saturado debajo del nivel freático, sobre el cual se tiene una franja capilar. Encima de la franja capilar, se sitúa una zona no saturada hasta la superficie del terreno.

TABLA 1.VALORES DE LOS PARÁMETROS GEOTÉCNICOS
E HIDROLÓGICOS EMPLEADOS EN LA MODELACIÓN
\begin{tabular}{c|c} 
Parámetro & Valor \\
\hline$I_{Z L T}(\mathrm{~m} / \mathrm{s})$ & $5,0 \times 10^{-7}$ \\
\hline$c^{\prime}\left(\mathrm{kN} / \mathrm{m}^{2}\right)$ & 9 \\
\hline$\varphi^{\prime}\left({ }^{\circ}\right)$ & 24 \\
\hline$\gamma_{s}\left(\mathrm{kN} / \mathrm{m}^{3}\right)$ & 15 \\
\hline$K_{s}(\mathrm{~m} / \mathrm{s})$ & $5,0 \times 10^{-5}$ \\
\hline$D_{0}\left(\mathrm{~m}^{2} / \mathrm{s}\right)$ & $1,0 \times 10^{-2}$ \\
\hline$\theta_{s}$ & 0,45 \\
\hline$\theta_{r}$ & 0,1 \\
\hline$a\left(\mathrm{~m}^{-1}\right)$ & 1 \\
\hline
\end{tabular}


De forma similar, se definen las variables requeridas en el modelo de interceptación de lluvia. Aunque los parámetros del modelo dependen de las especies de vegetación, densidad vegetativa, índice de área superficial, capacidad de almacenamiento de las hojas, ramas y tallos, entre otras propiedades, se realiza una única modelación de la interceptación para todos los escenarios de densidad arbórea simulados. La Tabla 2 muestra los valores adoptados para estas variables.

TABLA 2. VALORES DE LAS VARIABLES DEL MODELO DE INTERCEPTACIÓN DE LLUVIA

\begin{tabular}{c|c} 
Variable & Valor \\
\hline$b_{0}$ & 0,59 \\
\hline$C_{m}(\mathrm{~mm})$ & 0,78 \\
\hline $\bar{e}(\mathrm{~mm} / \mathrm{h})$ & 0,35 \\
\hline$k$ & 0,41
\end{tabular}

TABLA 3. VALORES DEL REFUERZO DE LAS RAÍCES (c) Y SOBRECARGA ARBÓREA $\left(m_{t}\right)$ PARA LOS ESCENARIOS MODELADOS: ESCENARIO 1: BAJA DENSIDAD ARBÓREA EN TODA LA CUENCA; ESCENARIO 2: ALTA DENSIDAD ARBÓREA EN TODA LA CUENCA; ESCENARIO 3: DENSIDAD ARBÓREA VARIABLE EN LA CUENCA

\begin{tabular}{c|c|c|}
\hline Escenario & $\left(\mathbf{k N} / \mathbf{m}^{2}\right)$ & $\left(\mathbf{k N} / \mathbf{m}^{\mathbf{2}}\right)$ \\
\hline 1 & 0,4 & 0,5 \\
\hline 2 & 2,0 & 1,8 \\
\hline \multirow{3}{*}{3} & Zona A: 0 & Zona A: 0 \\
\cline { 2 - 3 } & Zona B: 0,5 & Zona B: 0,4 \\
\cline { 2 - 3 } & Zona C: 0,9 & Zona C: 0,8 \\
& Zona D: 2,0 & Zona D: 1,8 \\
\hline
\end{tabular}

El refuerzo aportado por la raíces $\left(c_{r}\right)$ y la sobrecarga arbórea $\left(m_{t}\right)$ se modelaron en tres escenarios distintos, para los cuales varían los valores de $c_{r}$ y $m_{t}$ de acuerdo al caso de densidad arbórea que es simulado, con base en rangos de valores encontrados en la literatura (por ejemplo Hammond et al., 1992; J. E. Norris et al., 2008). Estos escenarios y los valores adoptados se muestran en la Tabla 3. En los escenarios 1 y 2 , se consideran valores de $c_{r}$ y $m_{t}$ constantes en toda la cuenca. En el primer escenario, se supone una baja densidad arbórea; en el segundo escenario, una alta densidad arbórea. Por su parte, el tercer escenario considera la variabilidad de la densidad arbórea sobre la cuenca mediante cuatro zonas en las que se tienen diferentes valores de $c_{r}$ y $m_{t^{\prime}}$ como se muestra en el mapa de la Figura 3.

Figura 3. Zonas diferenciadas por densidad arbórea en la cuenca. Zona A: sin presencia arbórea $\left(c_{r}=0, m_{t}=0\right)$. Zona B: baja densidad arbórea $\left(c_{r}=0,5 \mathrm{kN} / \mathrm{m}^{2}, m_{t}=0,4 \mathrm{kN} / \mathrm{m}^{2}\right)$. Zona C: densidad arbórea intermedia $\left(c=0,9 \mathrm{kN} / \mathrm{m}^{2}, m_{t}\right.$ $\left.=0,8 \mathrm{kN} / \mathrm{m}^{2}\right)$. Zona D: alta densidad arbórea $\left(c_{r}=2,0 \mathrm{kN} /\right.$ $\left.\mathrm{m}^{2}, m_{\mathrm{t}}=1,8 \mathrm{kN} / \mathrm{m}^{2}\right)$.

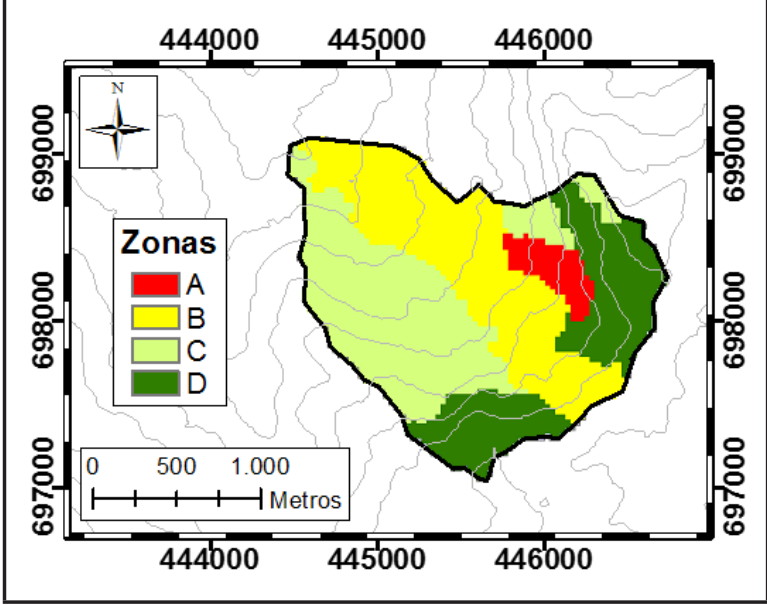

\section{RESULTADOS Y ANÁLISIS}

La lluvia simulada, obtenida tras evaluar la cantidad interceptada por el dosel arbóreo, se compara con la lluvia total en los hietogramas mostrados en la Figura 4. La lluvia total representa la tormenta de diseño que se utiliza en la simulación con TRIGRS 2.0, en la que no se considera ningún efecto de la presencia vegetal. Por su parte, la lluvia simulada constituye los datos de entrada para la modelación en la que se considera la interceptación de lluvia mediante el modelo de Liu (1997). En esta simulación, la cantidad total de lluvia interceptada durante toda la tormenta es de $2,1 \mathrm{~mm}$, lo que corresponde al 2,2\% de la precipitación total. Se observa que la mayor cantidad de agua interceptada se presenta en la primera hora del evento de lluvia, hasta que se reduce significativamente el índice de sequedad $(D)$. De esta manera, en los primeros treinta minutos se 
intercepta el $24,4 \%$ de lo que se intercepta en todo el evento de lluvia y en el segundo intervalo de tiempo (30-60 min) el 20,4\%.

Figura 4. Hietogramas de la lluvia total y de la lluvia simulada. Intensidad promedio $(\mathrm{mm} / \mathrm{h})$ en intervalos de tiempo de 30 minutos

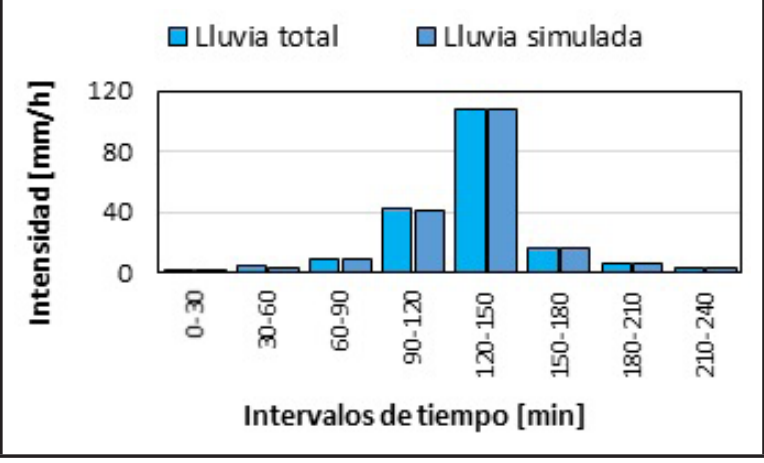

Cuando el índice de sequedad inicial se aproxima a cero (para los intervalos de tiempo definidos), es decir $D_{0} \approx 0$, la capacidad de almacenamiento casi que ha llegado a su capacidad máxima y el aporte de interceptación de lluvia se debe principalmente al efecto de evaporación, dado por la tasa de evaporación promedio del dosel ( $\bar{e}$ ). Por lo tanto, la interceptación de lluvia es muy baja en estos intervalos de tiempo.

Las Figuras 5 y 6 muestran el factor de seguridad (FS) en las cuatro simulaciones, antes y después del tiempo de duración $(4 \mathrm{~h})$ de sus tormentas de diseño. Las Figuras $\mathbf{5 a}$ y $\mathbf{b}$ muestran la modelación con TRIGRS 2.0, sin considerar el efecto de los árboles, desde el inicio de la tormenta ( $t=0$, Figura 5a) hasta el final de la misma ( $t=4 \mathrm{~h}$, Figura $\mathbf{5 b}$ ). De igual forma, las Figuras 5c y d presentan FS en el escenario 1 , donde se simula una baja densidad arbórea en toda la cuenca $\left(c_{r}=0,4 \mathrm{kN} / \mathrm{m}^{2}, m_{t}=0,5 \mathrm{kN} /\right.$ $\mathrm{m}^{2}$ ). Asimismo, las Figuras 5e y $\mathbf{f}$ muestran FS en el escenario 2, el cual representa una alta densidad arbórea a lo largo de la cuenca $\left(c_{r}=2,0 \mathrm{kN} / \mathrm{m}^{2}, m_{t}\right.$ $=1,8 \mathrm{kN} / \mathrm{m}^{2}$ ). Finalmente, la Figura 6 muestra FS al inicio y al final de la tormenta para el escenario 3, donde se tiene variabilidad espacial de la densidad arbórea sobre la cuenca.
En estas simulaciones, se predice que ocurre falla en las celdas en las cuales FS es menor que 1,0. En todas las modelaciones se observa una reducción de FS en múltiples celdas debido a la infiltración del agua de la lluvia. Asimismo, se presentan valores más altos de FS en los escenarios con mayor densidad arbórea; es decir, en los que se modela con valores más altos de $c_{r}$ y $m_{t^{\prime}}$ De esta manera, en la modelación sin considerar el aporte arbóreo (Figura 5a, b) se obtuvieron los valores más bajos de FS y se presentó el mayor número de celdas con $\mathrm{FS}<1$ (se predice falla en el 6,5\% del área de la cuenca). Por su parte, en el escenario en el cual se emplean valores relativamente bajos de $c_{r}$ y $m_{t^{\prime}}$ simulando baja densidad arbórea en toda la cuenca (Figura $\mathbf{5 c}, \mathbf{d}$ ), las celdas que fallan al término de la tormenta se disminuyen aproximadamente en la mitad (3,3\% del área de la cuenca con $\mathrm{FS}<1$ ), con respecto a la simulación en TRIGRS. En contraste, al simular una muy alta densidad arbórea a lo largo de toda la cuenca (Figura 5e, f), nuevamente al finalizar la tormenta se reducen los valores de FS; sin embargo, ninguna de las celdas falla.

Finalmente, en el escenario en el que se delimitan diferentes zonas de acuerdo a la presencia arbórea en áreas diferenciadas de la cuenca (Figura 6a, b), variando espacialmente los valores de $c_{r}$ y $m_{t^{\prime}}$ se obtienen pocas zonas que presentan inestabilidad al final de la tormenta (con 93 celdas en donde FS $<1$; es decir, el 0,3\% del área de la cuenca).

Al comparar las demás modelaciones con el último escenario (Figura 6), en donde $c_{r}$ y $m_{t}$ varían en la cuenca de acuerdo a la densidad arbórea de las diferentes zonas definidas, se evidencia la influencia de dicha delimitación en los resultados obtenidos. Todas las celdas en las que se predice falla en los escenarios de densidad arbórea simulados corresponden a áreas inestables en la modelación realizada con TRIGRS. En los escenarios 1 y 3 se predijo un 50,4\% y 4,9\%, respectivamente, de las áreas que fallan en la modelación con TRIGRS. Por otra parte, entre estos escenarios se observa que, si bien es muy pequeña el área inestable en el escenario 3 (densidad arbórea variable; 
Figura 6b), el 44,1\% de estas se predicen como áreas estables en el escenario 1 (baja densidad arbórea; Figura 5d). Esto se debe a que en algunas zonas con pendientes pronunciadas, en las cuales se tiene alta densidad arbórea (escenario 3), se tienen valores altos de $c_{r}$ y $m_{t}$ que contribuyen a la estabilidad; por su parte, en la mayor parte de terreno en la que hay muy baja densidad arbórea las pendientes no son tan pronunciadas como para que se produzcan deslizamientos superficiales.

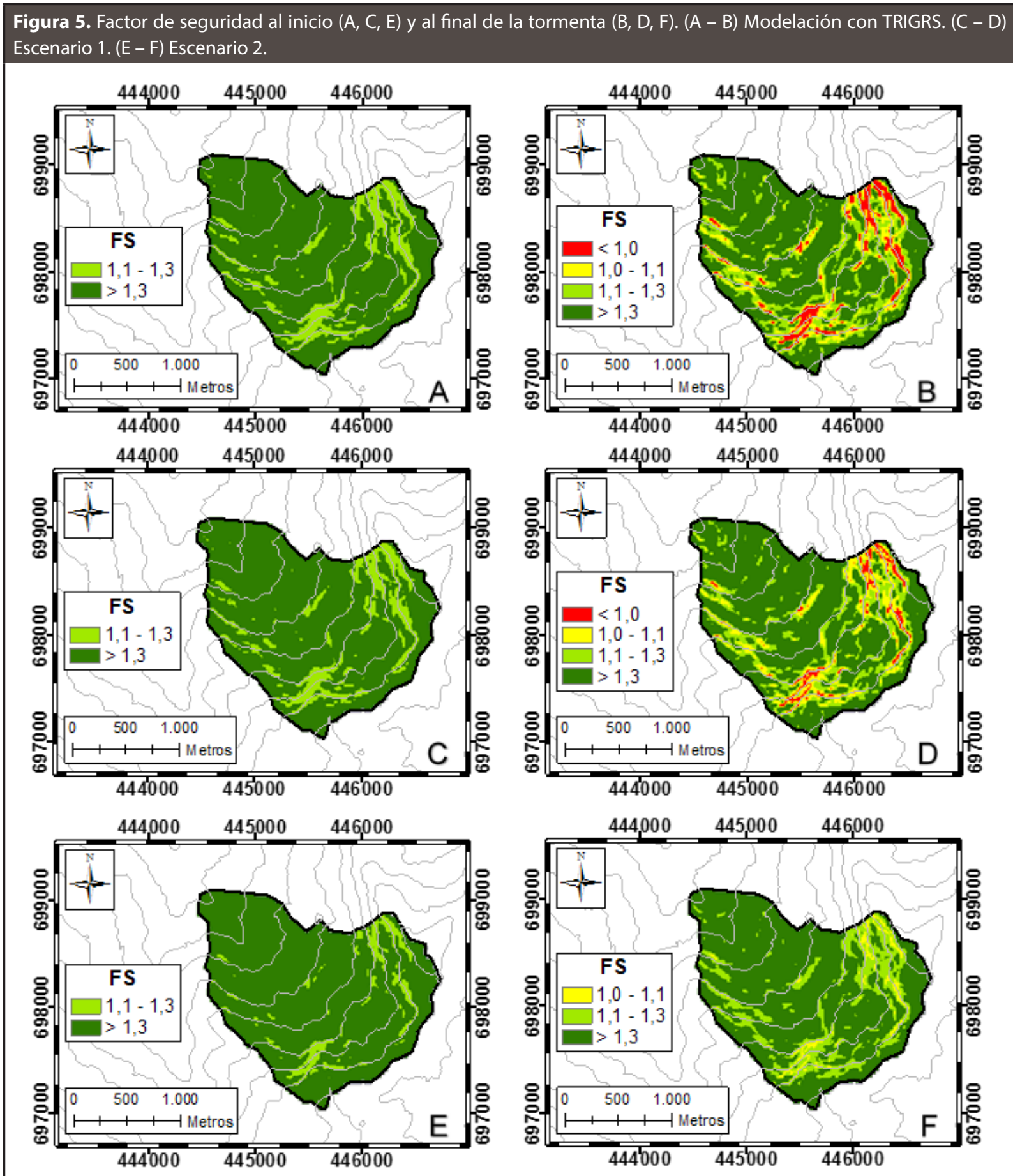


Figura 6. Factor de seguridad al inicio (A) y al final (B) de la tormenta. Escenario 3, con variabilidad espacial de la densidad arbórea sobre la cuenca. (A) $t=0 ;$ (B) $t=4 \mathrm{~h}$.
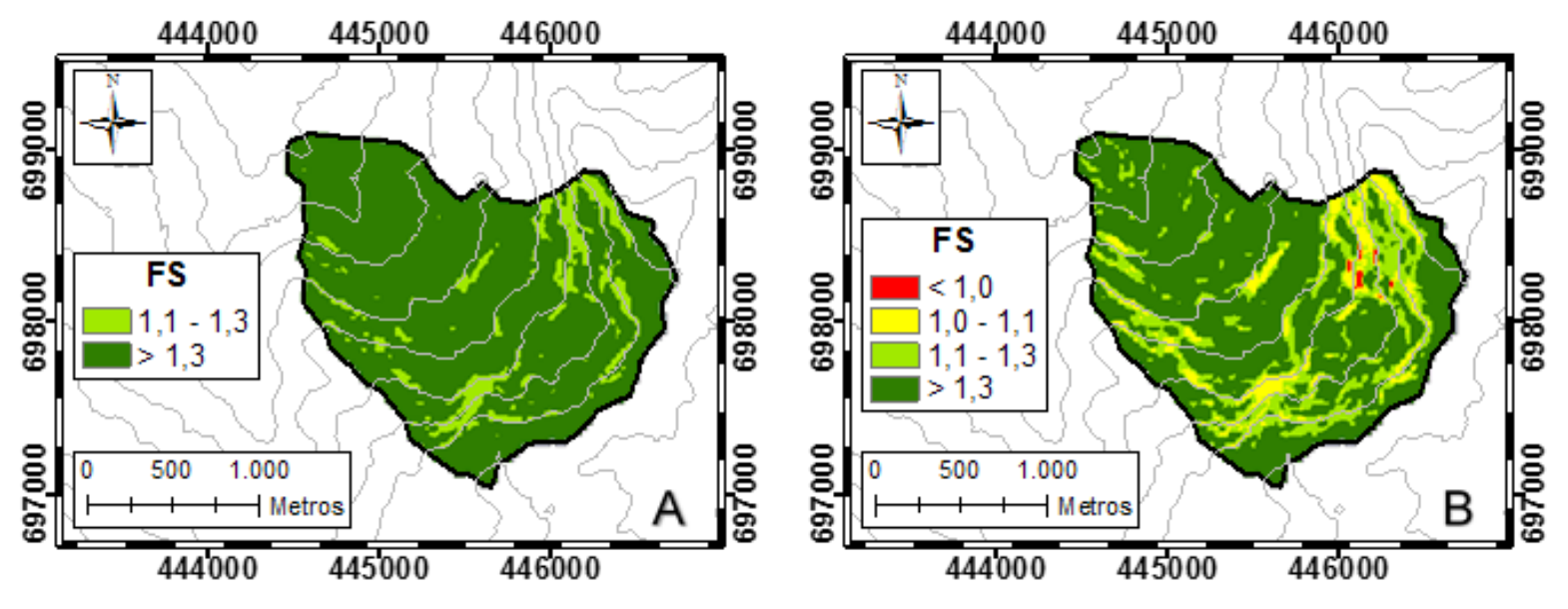

La posibilidad de delimitar pequeñas áreas dentro de la cuenca para asignar valores del refuerzo aportado por las raíces $\left(c_{r}\right)$ y la sobrecarga $\left(m_{t}\right)$, de acuerdo a la densidad arbórea observada u otra información adicional, permite simular la contribución arbórea de una manera más precisa a como se haría asignando valores constantes a estas variables. Los resultados mostrados indican que el método propuesto es muy sensible a los valores de $m_{t}$ y $c_{r}$, principalmente a este último. Una inspección visual de la Ecuación (8) muestra que en el presente análisis, $c_{r}$ puede considerarse como una cohesión adicional aportada por las raíces de los árboles. En otras investigaciones, en las cuales no se incluyen términos asociados al efecto de los árboles, se aumentan los valores de cohesión y ángulo de fricción para considerar el efecto de las raíces de los árboles en ciertas áreas (por ejemplo Chien-Yuan et al., 2005). En la utilización de TRIGRS se tiende a sobreestimar inestabilidades debido a la falta de información detallada de las propiedades topográficas y del suelo a lo largo del área estudiada (Jelínek y Wagner, 2007; Kim et al., 2010). Muchas veces la sobreestimación de deslizamientos, en modelos de este tipo, se debe a que en áreas con vegetación con sistemas de raíces que contribuyen a la estabilidad no se cuantifican sus efectos (Zizioli et al., 2013).

\section{CONCLUSIONES}

Se propone un método para analizar la susceptibilidad a ocurrencia de deslizamientos superficiales, a escala de cuenca, incluyendo el aporte de los árboles mediante tres parámetros principales: interceptación de lluvia, refuerzo de las raíces y sobrecarga. Se presenta el modelo de Liu (1997) como el modelo de interceptación de lluvia que permite determinar la cantidad de agua disponible para infiltración y su distribución temporal durante la duración de la misma. Así mismo, se describen los modelos hidrológicos de TRIGRS 2.0 para la simulación de la infiltración transitoria, de acuerdo a los tipos de condiciones iniciales que permite simular el modelo: saturadas y no saturadas, en los tipos de frontera basal (finita e infinita) que este admite. La presión de poros se calcula con el modelo de infiltración seleccionado y se emplea en el modelo revisado de estabilidad de taludes infinitos de Kim et al. (2013), el cual incluye los términos de refuerzo de las raíces y sobrecarga, y representa la estabilidad en términos del factor de seguridad (FS). FS se calcula para cada una de las celdas que en su conjunto constituyen la totalidad de la cuenca.

En la aplicación del método en una cuenca del Valle de Aburrá, se emplearon valores promedios 
para muchas de las variables geotécnicas e hidrológicas, las cuales en realidad varían espacialmente. En modelaciones a escala de cuenca son necesarias este tipo de simplificaciones debido a la dificultad para obtener información precisa de su variabilidad a lo largo del terreno. Sin embargo, información más precisa de estas variables mejoraría ampliamente los resultados obtenidos, por lo cual muchas de las investigaciones de este tipo suelen contar con información de campo mejor detallada. Esto mismo sucede con la resolución espacial (10 m x 10 m) del modelo digital de elevaciones empleado. Una mayor resolución espacial puede permitir la obtención de mejores resultados en la predicción de deslizamientos superficiales en este tipo de simulaciones (Montrasio, Valentino y Losi, 2011).

La simulación de diferentes escenarios de densidad arbórea y la modelación usando el modelo de TRIGRS permiten observar ciertas tendencias y resultados importantes. Por un lado, se obtuvo un valor pequeño de interceptación de lluvia $(2,1 \mathrm{~mm})$ en la simulación realizada, observando que en el modelo la interceptación no representa un componente significativo en la estabilidad, al evaluarse lluvias de alta intensidad y corta duración. En cambio, el refuerzo aportado por las raíces y la sobrecarga de los árboles tienen una mayor influencia en la estabilidad, de acuerdo a los resultados obtenidos, para las condiciones simuladas. La posibilidad de simular la variabilidad espacial de la densidad arbórea permite describir más adecuadamente las condiciones reales de campo y obtener mejores resultados en la modelación del aporte de los árboles a la estabilidad.

El método propuesto presenta básicamente las mismas desventajas potenciales que se reconocen en el modelo revisado de Kim et al. (2013), al igual que otros métodos que emplean un modelo de estabilidad de talud infinito para la predicción de deslizamientos superficiales. La principal de ellas es que estos modelos aplican para deslizamientos superficiales planares y no para movimientos en masa profundos o con fallas circulares. Por su parte, el método descrito se diferencia en que el modelo de interceptación de lluvia empleado utiliza únicamente parámetros físicos (ningún parámetro empírico) con significados físicos específicos. Igualmente, difiere en que los modelos de infiltración de TRIGRS 2.0, que se proponen en nuestro método, son aplicables a un rango más amplio de condiciones iniciales. Si bien en el modelo revisado de Kim et al. (2013) se utiliza el modelo hidrológico de la primera versión de TRIGRS (Baum, Savage y Godt, 2002), en su modelo es perfectamente aplicable el modelo hidrológico de TRIGRS 2.0.

Para estudiar de forma más profunda los efectos de los árboles en la estabilidad de taludes, se requieren llevar a cabo más investigaciones de los mecanismos que influyen en la estabilidad, así como realizar modelaciones en diferentes condiciones topográficas y eventos de lluvia. Por su parte, el método propuesto requiere ser aplicado con estimaciones más precisas de las variables y un evento de lluvia real que haya causado movimientos en masa en las laderas de una cuenca, de tal manera que las condiciones reales sean simuladas de la forma más aproximada posible y los resultados se puedan comparar con registros existentes del fenómeno.

\section{REFERENCIAS}

Baum, R. L.; Savage, W. Z.; Godt, J. W. (2002). TRIGRS-a Fortran program for transient rainfall infiltration and grid-based regional slope-stability analysis. USGS Open File Report 02-0424. US Geological Survey, Reston, VA.

Baum, R. L.; Savage, W. Z.; Godt, J. W. (2008). TRIGRS- $A$ Fortran Program for Transient Rainfall Infiltration and Grid-Based Regional Slope-Stability Analysis, Version 2. 0. U.S. Geological Survey Open-File Report.

Baum, R. L.; Godt, J. W.; Savage, W. Z. (2010). Estimating the timing and location of shallow rainfall-induced landslides using a model for transient, unsaturated infiltration. Journal of Geophysical Research: Earth Surface, 115(F3).

Baum, R. L.; Godt, J. W.; Coe, J. A. (2011). Assessing susceptibility and timing of shallow landslide and debris flow initiation in the Oregon Coast Range, 
USA. In 5th International Conference on Debris-Flow Hazards Mitigation: Mechanics, Prediction, and Assessment, pp. 825-834.

Bordoni, M.; Meisina, C.; Valentino, R.; Bittelli, M.; Chersich, S. (2015a). Site-specific to local-scale shallow landslides triggering zones assessment using TRIGRS. Natural Hazards \& Earth System Sciences, 15(5).

Bordoni, M.; Meisina, C.; Valentino, R.; Lu, N.; Bittelli, M.; Chersich, S. (2015b). Hydrological factors affecting rainfall-induced shallow landslides: From the field monitoring to a simplified slope stability analysis. Engineering Geology, 193, pp. 19-37.

Burton, A.; Bathurst, J. C. (1998). Physically based modelling of shallow landslide sediment yield at a catchment scale. Environmental Geology, 35(2-3), pp. 89-99. Disponible en: http://doi.org/10.1007/ s002540050296.

Cascini, L.; Cuomo, S.; Della Sala, M. (2011). Spatial and temporal occurrence of rainfall-induced shallow landslides of flow type: A case of Sarno-Quindici, Italy. Geomorphology, 126(1), pp. 148-158.

Chien-Yuan, C.; Tien-Chien, C.; Fan-Chieh, Y.; Sheng-Chi, L. (2005). Analysis of time-varying rainfall infiltration induced landslide. Environmental Geology, 48(4-5), pp. 466-479.

Coe, J. A.; Michael, J. A.; Crovelli, R. A.; Savage, W. Z.; Laprade, W. T.; Nashem, W. D. (2004). Probabilistic assessment of precipitation-triggered landslides using historical records of landslide occurrence, Seattle, Washington. Environmental \& Engineering Geoscience, 10(2), pp. 103-122.

Chow, V. T.; Maidment, D. R.; Mays, L. W. (1988). Applied hydrology. McGraw-Hill Publishing Company, 572 p.

Crovelli, R. A. (2000). Probability models for estimation of number and costs of landslides. US Geological Survey.

Frattini, P.; Crosta, G.; Sosio, R. (2009). Approaches for defining thresholds and return periods for rainfalltriggered shallow landslides. Hydrological Processes, 23(10), pp. 1444-1460. Disponible en: http:// doi.org/10.1002/hyp.7269.

Godt, J. W.; Baum, R. L.; Chleborad, A. F. (2006). Rainfall characteristics for shallow landsliding in Seattle, Washington, USA. Earth Surface Processes and Landforms, 31(1), pp. 97-110.
Gray, D. H.; Sotir, R. B. (1996). Biotechnical and soil bioengineering slope stabilization: A practical guide for erosion control. John Wiley \& Sons, Ed. Wiley, pp. 101-102.

Guzzetti, F.; Galli, M.; Reichenbach, P.; Ardizzone, F.; Cardinali, M. (2006). Landslide hazard assessment in the Collazzone area, Umbria, Central Italy. Natural Hazards and Earth System Science, 6(1), pp. 115-131.

Guzzetti, F.; Peruccacci, S.; Rossi, M.; Stark, C. P. (2008). The rainfall intensity-duration control of shallow landslides and debris flows: an update. Landslides, 5(1), pp. 3-17.

Hammond, C.; Hall, D.; Miller, S.; Swetic, P. (1992). Level I Stability Analysis (LISA) documentation for version 2.0. USDA Forest Service Intermountain Research Station, Ogden, UT, General Technical Report INT285. US Department of Agriculture, Forest Service, Intermountain Research Station, pp. 30-32.

ISEA Ltda. (2006). Plan de saneamiento y manejo de vertimientos - PSMV - Vereda el Cabuyal, municipio de Copacabana-Antioquia, $182 \mathrm{p}$.

Iverson, R. M. (2000). Landslide triggering by rain infiltration. Water Resources Research, 36(7), pp. 1897-1910. Disponible en: http://doi. org/10.1029/2000WR900090.

Jelínek, R.; Wagner, P. (2007). Landslide hazard zonation by deterministic analysis (Vel'ká Causa landslides area, Slovakia). Lanslides, 4(4), pp. 339-350.

Kim, D.; Im, S.; Lee, S. H.; Hong, Y.; Cha, K.-S. (2010). Predicting the rainfall-triggered landslides in a forested mountain region using TRIGRS model. Journal of Mountain Science, 7(1), pp. 83-91.

Kim, D.; Im, S.; Lee, C.; Woo, C. (2013). Modeling the contribution of trees to shallow landslide development in a steep, forested watershed. Ecological Engineering, 61, pp. 658-668. Disponible en: http://doi. org/10.1016/j.ecoleng.2013.05.003.

Liao, Z.; Hong, Y.; Kirschbaum, D.; Adler, R. F.; Gourley, J. J.; Wooten, R. (2011). Evaluation of TRIGRS (transient rainfall infiltration and grid-based regional slope-stability analysis)'s predictive skill for hurricane-triggered landslides: a case study in Macon County, North Carolina. Natural Hazards, 58(1), pp. 325-339.

Revista EIA Rev.ElA.Esc.Ing.Antioq / Universidad EIA 
Liu, C.-N.; Wu, C.-C. (2008). Mapping susceptibility of rainfall-triggered shallow landslides using a probabilistic approach. Environmental Geology, 55(4), pp. 907-915.

Liu, S. (1997). A new model for the prediction of rainfall interception in forest canopies. Ecological Modelling, 99(2-3), pp. 151-159. Disponible en: http:// doi.org/10.1016/S0304-3800(97)01948-0.

Marín, R. J.; Castro, J. D. (2015). Efecto de los árboles en la ocurrencia de deslizamientos superficiales en una cuenca del Valle de Aburrá. Universidad de Antioquia, $103 \mathrm{p}$.

Meisina, C.; Scarabelli, S. (2007). A comparative analysis of terrain stability models for predicting shallow landslides in colluvial soils. Geomorphology, 87(3), pp. 207-223.

Montgomery, D. R.; Dietrich, W. E. (1994). A physically based model for the topographic control on shallow landsliding. Water Resources Research, 30(4), pp. 1153-1171.

Montrasio, L.; Valentino, R.; Losi, G. L. (2011). Towards a real-time susceptibility assessment of rainfall-induced shallow landslides on a regional scale. Natural Hazards and Earth System Science, 11(7), pp. 1927-1947.

Montrasio, L.; Schilirò, L.; Terrone, A. (2015). Physical and numerical modelling of shallow landslides. Landslides, pp. 1-11.

Morgan, R. P. C.; Rickson, R. J. (2003). Slope Stabilization and Erosion Control: A Bioengineering Approach: A Bioengineering Approach. Taylor \& Francis. pp. 3740.

Norris, J. E., Greenwood, J. R., Achim, A., Gardiner, B. A., Nicoll, B. C., Cammeraat, E., \& Mickovski, S. B. (2008). Hazard assessment of vegetated slopes. In Slope Stability and Erosion Control: Ecotechnological Solutions, Springer, pp. 119-166.

Pack, R. T.; Tarboton, D. G.; Goodwin, C. N. (1998). The SINMAP approach to terrain stability mapping. In 8 th congress of the international association of engineering geology, Vancouver, British Columbia, Canada, pp. 21-25.

Park, D. W.; Nikhil, N. V.; Lee, S. R. (2013). Landslide and debris flow susceptibility zonation using TRIGRS for the 2011 Seoul landslide event. Natural Hazards and Earth System Sciences, 13(11), pp. 2833-2849.

Raia, S.; Alvioli, M.; Rossi, M.; Baum, R. L.; Godt, J. W.; Guzzetti, F. (2013). Improving predictive power of physically based rainfall-induced shallow landslide models: a probabilistic approach. Geoscientific Model Development and Discussions, 6(1), pp. 1367-1426.

Richards, L. A. (1931). Capillary conduction of liquids through porous mediums. Journal of Applied Physics, 1(5), pp. 318-333.

Rutter, A. J. J.; Kershaw, K. A. A.; Robins, P. C. C.; Morton, A. J. J. (1971). A predictive model of rainfall interception in forests, 1. Derivation of the model from observations in a plantation of Corsican pine. Agricultural Meteorology, 9(1969), pp. 367-384. Disponible en: http://doi.org/10.1016/0002-1571(71)90034-3.

Rutter, A. J.; Morton, A. J.; Robins, P. C. (1975). A predictive model of rainfall interception in forests. II. Generalization of the model and comparison with observations in some coniferous and hardwood stands. Journal of Applied Ecology, pp. 367-380.

Salciarini, D.; Godt, J. W.; Savage, W. Z.; Conversini, P.; Baum, R. L.; Michael, J. A. (2006). Modeling regional initiation of rainfall-induced shallow landslides in the eastern Umbria Region of central Italy. Landslides, 3(3), pp. 181-194.

Sidle, R. C.; Ochiai, H. (2006). Landslides: Processes, Prediction, and Land Use. American Geophysical Union, pp. 89-110.

Smith, R.; Vélez, M. V. (1997). Hidrología de Antioquia. Medellín: Secretaría de Obras Públicas Departamentales, $253 \mathrm{p}$.

Stokes, A.; Norris, J.; van Beek, L. P. H.; Bogaard, T.; Cammeraat, E.; Mickovski, S.; Fourcaud, T. (2008). How Vegetation Reinforces Soil on Slopes. In J. Norris, A. Stokes, S. Mickovski, E. Cammeraat, R. van Beek, B. Nicoll, \& A. Achim (Eds.), Slope Stability and Erosion Control: Ecotechnological Solutions SE - 4, pp. 65118. Springer Netherlands. Disponible en: http:// doi.org/10.1007/978-1-4020-6676-4_4.

Taylor, D. W. (1948). Fundamentals of soil mechanics. Soil Science, 66(2), $161 \mathrm{p}$.

Vieira, B. C.; Fernandes, N. F. (2010). Shallow landslide prediction in the Serra do Mar, Sao Paulo, Brazil. 
Natural Hazards and Earth System Sciences, 10(9), pp. 1829-1837.

Wu, W.; Sidle, R. C. (1995). A distributed slope stability model for steep forested basins. Water Resources Research, 31(8), pp. 2097-2110.

Zizioli, D.; Meisina, C.; Valentino, R.; Montrasio, L. (2013). Comparison between different approaches to modeling shallow landslide susceptibility: a case history in Oltrepo Pavese, Northern Italy. Nat Hazards Earth Syst Sci, 13(3), pp. 559-573.

Marín, R. J.; Osorio, J. P. (2017). Modelación de la contribución arbórea en análisis de susceptibilidad a deslizamientos superficiales. Revista EIA, 14(28), julio-diciembre, pp. 1328. [Online]. Disponible en: https://doi.org/10.24050/reia. v14i28.975 